\title{
Verzeichnis der abgekürzt zitierten Literatur
}

\author{
A/D/S 6 \\ $A / F / R$ \\ $B / B$ \\ $\mathrm{BeBiKo}{ }^{11}$ \\ BeckOK \\ B/H GmbHG ${ }^{21}$ \\ B/H HGB ${ }^{38}$ \\ Binz/Sorg ${ }^{11}$ \\ Blümich \\ Bordewin/Brandt \\ $\mathrm{B} / \mathrm{R} / \mathrm{O}^{7}$ \\ BStGB \\ Crezelius $L B^{2}$ \\ $D / P / M$ \\ E/H \\ Ernst \& Young \\ E/S KStG ${ }^{3}$ \\ F/M \\ F/P/G \\ Frotscher/Geurts \\ Fuhrmann ${ }^{3}$ \\ F/W/B/S \\ $F / W / K$ \\ Gl/Gür GewSt $G^{9}$ \\ Gl $H_{G B}{ }^{7}$ \\ G/K/G/K \\ Gosch \\ Gosch ${ }^{3}$ \\ Helmke/Bauer \\ $H / H / R$ \\ $H / H / S p$ \\ $H / M / W$ \\ Höfer BetrAVG ArbR \\ Hüttemann $^{4}$ \\ $\mathrm{H} / \mathrm{V} / \mathrm{V}$ \\ $H / W$ \\ Jakob $L B^{4}$ \\ Jauernig $^{17}$ \\ K/F/K \\ Kirchhof/Ratschow \\ Adler/Düring/Schmaltz, Rechnungslegung und Prüfung der Unternehmen, \\ 6. Aufl. 2000 \\ Ahrend/Förster/Rößler, Steuerrecht der betrieblichen Altersversorgung \\ (Losebl.) \\ Bordewin/Brandt, Kommentar zum Einkommensteuergesetz (Losebl.) \\ Beck'scher Bilanz-Kommentar, 11. Aufl. 2018 \\ Beck'scher Online-Kommentar \\ Baumbach/Hueck, GmbH-Gesetz, Kommentar, 21. Aufl. 2017 \\ Baumbach/Hopt, Handelsgesetzbuch, Kommentar, 38. Aufl. 2018 \\ Binz/Sorg, GmbH \& Co. KG, 11. Aufl. 2010 \\ Blümich, Kommentar zu EStG, KStG, GewStG und Nebengesetzen (Losebl.) \\ Bordewin/Brandt, Einkommensteuergesetz, Kommentar (Losebl.) \\ Blomeyer/Rolfs/Otto, Betriebsrentengesetz, 7. Aufl. 2018 \\ Bundessteuergesetzbuch - Ein Reformentwurf zur Erneuerung des Steuerrechts, \\ vorgelegt von Paul Kirchhof, 2011 \\ Crezelius, Steuerrecht II, 2. Aufl. 1994 \\ Dötsch/Pung/Möhlenbrock, Die Körperschaftsteuer, Kommentar zum KStG, \\ UmwStG und zu den einkommensteuerrechtlichen Vorschriften der Anteilse- \\ ignerbesteuerung (Losebl.) \\ Ebling/Heuermann, Die Kindergeldauszahlung durch den Arbeitgeber, 1996 \\ Ernst \& Young, Körperschaftsteuergesetz-Kommentar (Losebl.) \\ Erle/Sauter, Körperschaftsteuergesetz, Kommentar, 3. Aufl. 2010 \\ Frotscher/Maas, Kommentar zum Körperschaftsteuer- und Umwandlungs- \\ steuergesetz (Losebl.) \\ Felsmann/Pape/Giere/König, Einkommensbesteuerung der Land- und Forst- \\ wirte (Losebl.) \\ Frotscher/Geurts, Kommentar zum Einkommensteuergesetz (Losebl.) \\ Fuhrmann, Außensteuergesetz, Kommentar, 3. Aufl. 2017 \\ Flick/Wassermeyer/Baumhoff/Schönfeld, Kommentar zum Außensteuerrecht \\ (Losebl.) \\ Flick/Wassermeyer/Kempermann, DBA Deutschland-Schweiz, Kommentar \\ (Losebl.) \\ Glanegger/Güroff, Kommentar zum GewStG, 9. Aufl. 2017 \\ Glanegger/Kirnberger/Kusterer/Ruß/Selder/Stuhlfelner, Handelsgesetzbuch, \\ Kommentar, 7. Aufl. 2007 \\ Gosch/Kroppen/Grotherr/Kraft, DBA-Kommentar (Losebl.) \\ Gosch, Abgabenordnung, Finanzgerichtsordnung (Losebl.) \\ Gosch, Körperschaftsteuergesetz, Kommentar, 3. Aufl. 2015 \\ Familienleistungsausgleich (Losebl.) \\ Herrmann/Heuer/Raupach, Kommentar zum Einkommensteuer- und Körper- \\ schaftsteuergesetz (Losebl.) \\ Hübschmann/Hepp/Spitaler, Kommentar zur Abgabenordnung und Finanz- \\ gerichtsordnung (Losebl.) \\ Hartz/Meeßen/Wolf, ABC-Führer Lohnsteuer (Losebl.) \\ Höfer, Gesetz zur Verbesserung der betrieblichen Altersversorgung, Band I, \\ Arbeitsrecht (Losebl.) \\ Hüttemann, Gemeinnützigkeits- und Spendenrecht, 4. Aufl. 2018 \\ Höfer/Veit/Verhuven, Betriebsrentenrecht (BetrAVG) Band II: Steuerrecht/ \\ Sozialabgaben, HGB/IFRS (Losebl.) \\ Heuermann/Wagner, Lohnsteuer (Losebl.) \\ Jakob, Einkommensteuer, 4. Aufl. 2008 \\ Jauernig, Bürgerliches Gesetzbuch, Kommentar, 17. Aufl. 2018 \\ Klein/Flockermann/Kühr, Handbuch des Einkommensteuerrechts (Losebl.) \\ Kirchhof/Ratschow, Beck'scher Online-Kommentar zum Einkommensteuer- \\ gesetz, 2018
}




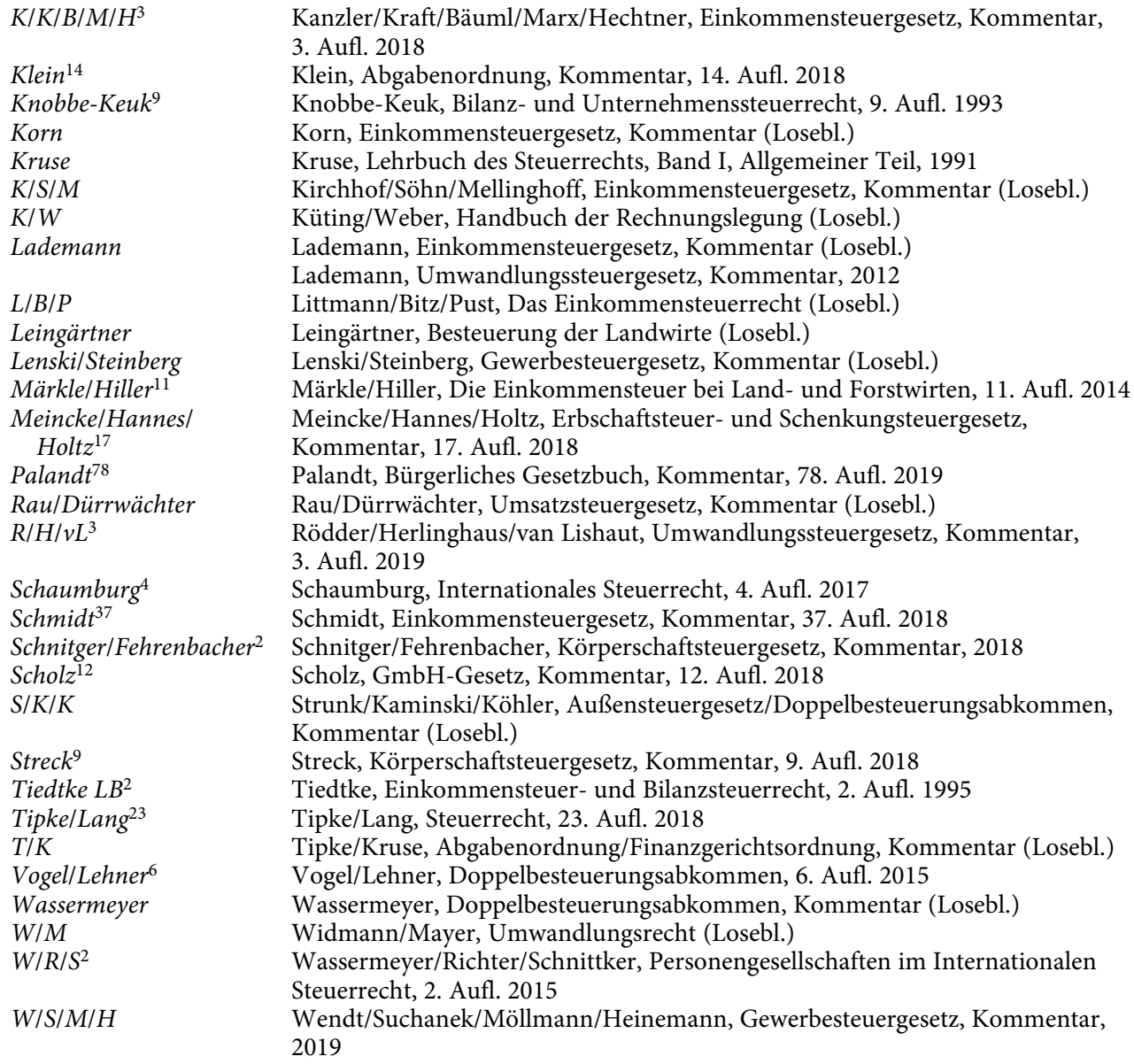

